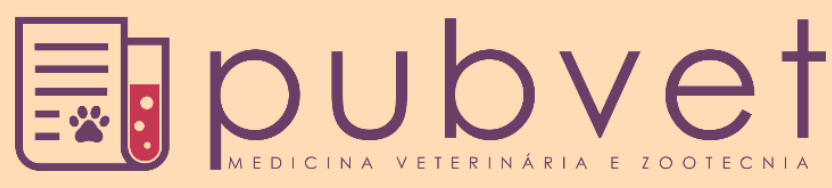

https://doi.org/10.31533/pubvet.v13n10a421.1-11

\title{
Potencial de fecundação diária de Touros Nelore na monta a campo selecionados por avaliações andrológicas e comportamento sexual
}

\author{
Daniel Fábio Salvador ${ }^{*} \bullet$, Luiz Altamiro Garcia Nogueira ${ }^{2} \bullet$, Venício José de Andrade A $^{\circ}$ \\ ${ }^{I}$ Professor Fundação CECIERJ, Rio de Janeiro - RJ, Brasil. \\ ${ }^{2}$ Professor Universidade Federal Fluminense, Niterói - RJ, Brasil. \\ ${ }^{3}$ Professor aposentado, Universidade Federal Minas Gerais, Belo Horizonte - MG, Brasil. \\ *Autor para correspondência, E-mail: salvador@cecierj.edu.br
}

Resumo. Foi avaliado o desempenho de touros Nelore em cobertura a campo por 4 dias com alta pressão de cios sincronizados, quanto a sua classificação andrológica por pontos(CAP) e teste de libido e testados em 4 tratamentos: $\mathrm{T} 1=\mathrm{CAP}>75$ pontos e alta libido; $\mathrm{T} 2=\mathrm{CAP}<75$ pontos e alta libido; $\mathrm{T} 3=\mathrm{CAP}>75$ e baixa libido; $\mathrm{T} 4=\mathrm{CAP}<75$ pontos e baixa libido. Os cios foram observados de 18 a 78 horas após a sincronização com os maiores percentuais registrados às $42(40,1 \%)$ e às $54(30,4 \%)$ horas. Foram registrados, em média, 24,62 $\pm 2,11$ fêmeas em cio por lote com pico de 9,87 $\pm 1,55$ fêmeas/touro/dia. O número médio de fêmeas gestante por touro foi de $9,25 \pm 3,2$. A taxa média de gestação foi de $33,33 \%$, variando de 22,44 no T4 a $47,82 \%$ no T1 (p<0,05). Observaram-se mais altas taxas com 42 horas $(42,67 \%)$ após a retirada os implantes, com destaque para o T2 com $52,38 \%$ de prenhes, porém com as menores taxas nos períodos subsequentes, quando comparado aos demais tratamentos. Embora fosse constatada diminuição geral nas taxas de prenhes às 54 horas, após a retirada dos implantes, os touros do T1 mantiveram as maiores taxas em relação aos demais tratamentos. Conclui-se que agregando CAP e teste de libido, pode-se compreender potencial de monta para touros Nelore frente a alto índice de manifestação de cios, permitindo assim maior pressão de seleção e melhor investimento na aquisição de touros de alto valor genético.

Palavras chave: Andrologia, CAP, desempenho reprodutivo, libido, Nelore, Zebu.

\section{Nelore bulls daily fertilization potential in the field selected by andrological assessments and sexual behavior}

\begin{abstract}
The performance of Nelore bulls in field was evaluated in single mating for 4 days with a large number of synchronized females for Nelore bulls with excellent andrological evaluation (BSE) and tested for libido were divided in 4 treatments: $\mathrm{T} 1=\mathrm{BSE}$ $>75$ points and high libido; $\mathrm{T} 2=\mathrm{BSE}<75$ points and high libido; $\mathrm{T} 3=\mathrm{BSE}>75$ and low libido, and T4 = BSE $<75$ points and low libido. Heat observation was done from 18 to 78 hours after implant removal, with the largest heat rates at $42(40.1 \%)$ and at $54(30.4 \%)$ hours. It was registered in average $24.62 \pm 2.11$ females in heat per group and estrus during the heat manifestation peak of $9.87 \pm 1.55$ females/bull. The pregnant female/bull mean rate during the challenge period was $9.25 \pm 3.20$. The pregnancy rate mean at the end of the 4 day challenge period was $33.33 \%$, ranging from $22.44 \%$ for T4 and $47.82 \%$ for T1 $(\mathrm{p}<0.05)$. The highest mean pregnancy rate was recorded 42 hours $(42.67 \%)$ after implant removal, with T2 showing the highest pregnancy rate $(52.38 \%)$ when compared to the other treatments, however with the lowest rates in the subsequent periods. Even though it was recorded decreasing in the general pregnancy rates at 54 hours after implant removal T1 bulls kept the higher pregnancy rates when compared to the other treatments.
\end{abstract}


Conclusions shows that putting together bulls with high BSE and libido scores, a higher number of cows can be served during breeding season with no commitment of reproductive efficiency, besides allowing higher selection pressure and lower economical investments in bulls with higher genetic value.

Keywords: Andrology, BSE, libido, Nelore bulls, reproductive performance, Zebu

\title{
Potencial de fecundación diaria de toros Nelore en la monta a campo seleccionados por evaluación andrológica y comportamiento sexual
}

\begin{abstract}
Resumen. Se evaluó el rendimiento de los toros Nelore en la monta a campo durante 4 días con alta presión de celos sincronizadas para su clasificación de puntos andrológicos (CAP) y prueba de libido, y se probó en 4 tratamientos: $\mathrm{T} 1=\mathrm{CAP}>75$ puntos y alta libido $\mathrm{T} 2=$ CAP $<75$ puntos y alta libido; T3 $=$ CAP $>75$ y baja libido; $\mathrm{T} 4=\mathrm{CAP}<75$ puntos y baja libido. Los celos se observaron de 18 a 78 horas después de la sincronización con los porcentajes más altos registrados a las $42(40,1 \%)$ y $54(30,4 \%)$ horas. Se registró un promedio de $24.62 \pm 2.11$ hembras en celo por lote con un pico de $9.87 \pm 1.55$ hembras/toro/día. El número promedio de hembras preñas por toro fue de $9.25 \pm 3.2$. La tasa promedio de embarazo fue del $33.33 \%$, que oscila entre 22.44 en T4 y $47.82 \%$ en T1 ( $\mathrm{p}<0.05$ ). Se observaron tasas más altas a las 42 horas $(42.67 \%$ ) después de la extracción de los implantes, especialmente $\mathrm{T} 2$ con $52.38 \%$ de vacas embarazadas, pero con las tasas más bajas en períodos posteriores, en comparación con otros tratamientos. Aunque hubo una disminución general en las tasas de embarazo a las 54 horas después de la extracción del implante, los toros T1 mantuvieron las tasas más altas en comparación con otros tratamientos. Se concluyó que, al agregar prueba de CAP y prueba de libido, es posible comprender mejor el potencial de reproducción de los toros Nelore frente a una alta tasa de manifestación del estro, lo que permite una mayor presión de selección y una mejor inversión en la adquisición de toros de alto valor genético.
\end{abstract}

Palabras clave: Andrología, CAP, desempeño reproductivo, libido, Nelore, Cebú

\section{Introdução}

A capacidade de fecundação diária de touros, bem como o potencial de fertilização a campo frente a desafios variados de fêmeas em cio, são questões fundamentais dentro do contexto da pecuária de corte, em virtude da escolha de adequadas razões entre touros e vacas, com reflexos diretos na eficiência reprodutiva, produtiva e econômica do rebanho.

O exame andrológico completo de touros, que potencialmente serão usados em monta natural, inclui as avaliações, clínica e da biometria testicular, das características espermáticas (aspectos físicos e morfológicos do sêmen), além da libido e da capacidade de monta, que são fatores altamente ligados à fertilidade e ao melhoramento genético de rebanhos bovinos (Costa e Silva, 1994). Resultados verificados em trabalhos conduzidos por vários autores (Costa e Silva, 1994; Fonseca et al., 1997; Franco et al., 2006; Freneau et al., 2017; Pineda et al., 2000; Pineda et al., 1997; Vale Filho, 1994) demonstraram o quanto os touros Nelore vêm sendo subtilizados, quando a razão média touro:vaca é de 1:25; porém, ainda existe escassez de relatos de caso e definições de qual seria o limite máximo de acasalamento de touros Nelore na monta a campo para condições extensivas (Salvador et al., 2001).

A classificação andrológica por pontos (CAP), sugerida por Vale Filho (1988) para as raças zebuínas, baseia-se nos moldes do "Breeding Soundness Evaluation" (BSE) proposto por Chenoweth (1983), com adaptações para touros Bos taurus indicus, quanto à idade na qual deverá ser computada a CE. Vale Filho (1988) sugere o dobro da idade para a mesma circunferência escrotal, proposta para Bos taurus taurus, destacando o fato de que, dependendo do nível nutricional, tais medidas somente serão alcançadas a uma idade ainda mais elevada. Quanto aos aspectos físicos e morfológicos do sêmen, estes seriam pontuados segundo os mesmos critérios sugeridos para os taurinos. A classificação andrológica por pontos (CAP) em touros Nelore revelou-se de grande utilidade para a seleção de touros a campo, sendo registrada uma alta herdabilidade para esta característica $(0,75)$, destacando a capacidade de 
seleção de touros para alto desempenho reprodutivo, baseada na CAP (Quirino et al., 2001; Vale Filho et al., 1999).

Em estudo sobre evolução por quatro anos da capacidade andrológica por pontos (CAP) de touros Nelore e sua relação com a porcentagem de prenhez em estação de monta, Freneau et al. (2017) observaram correlações entre o CAP com os seus componentes, o ano e as características reprodutivas estudadas. Houve correlação $(P<0,001)$ entre o CAP e a taxa de prenhez $(r=0,25)$, ou seja, utilização de touros de alta CAP favoreceu o aumento da percentagem de prenhez no rebanho, mostrando que a razão touro:fêmea observada subutiliza o potencial reprodutivo dos touros. O comportamento sexual de touros tem sido avaliado pela intensidade da libido (Costa e Silva, 1994; Rupp et al., 1977; Smith et al., 1981; Vale Filho, 1994) da capacidade de serviço (Blockey, 1978; Chenoweth et al., 1984; Rupp et al., 1977) e do comportamento sexual a campo para touros da raça Nelore(Santos et al., 2003). Trabalhando com touro europeus em sistema de acasalamento individual e múltiplo, com lotes de 25,40 e 60 fêmeas, numa estação de monta de 21 dias, Rupp et al. (1977) não constaram diferenças significativas quando a fertilidade entre os grupos estudados. Avaliando touros Angus, Polled Hereford e Santa Gertrudis, em lotes com 40 ou 50 fêmeas em estação de monta de 90 dias, Neville Júnior et al. (1988) não registraram diferenças entre os grupos e raças, observando ainda que o custo de manutenção de touros pode ser reduzido pela adequada utilização dos mesmos.

Usando o procedimento de regressão "stepwise", e tendo como variável dependente a taxa de prenhez das fêmeas em cio, durante quatro dias de desafio, Smith et al. (1981) observaram que a libido, os defeitos espermáticos secundários e a classificação no BSE, explicavam $44 \%$ da taxa de prenhez (P < $0,05)$, em touro Santa Gertrudis. No entanto, os autores concluíram que os métodos atuais de avaliação da fertilidade não têm acurácia adequada para predizer a fertilidade de touros submetidos a elevados desafios de acasalamento. Em condições brasileiras, Crudeli (1990) trabalhando com touros Nelore, com CE variando 33 a $37 \mathrm{~cm}$ e com capacidade de serviço alta, média e baixa, com estação de monta de 120 dias, não encontrou efeito $(\mathrm{P}>0,05)$ entre os grupos no teste de fertilidade a campo, atribuindo o insucesso à baixa razão touro:vaca utilizada (1:40), a qual não foi suficiente para desafiar aqueles touros com alta classificação nos exames andrológicos e comportamentais.

Usando o sistema de classificação de touros pontuando os animais quanto ao libido, Costa e Silva (1994) desafiou touros Nelore na proporção de 1:40 e 1:60 em acasalamentos individualizados. A classificação andrológica, a libido e a razão touro:vaca não influenciaram as taxas de gestação alcançadas. A autora sugerem que a não observância de diferenças não estavam relacionadas à inadequações nos sistemas de classificação e avaliação da libido para a raça Nelore e sim as baixas razões entre touros e vacas (1:40 e 1:60) utilizadas, que não proporcionaram suficiente desafio da capacidade fecundante dos touros, para que se pudesse identificar os animais superiores.

Conduzindo estudos com tourinhos Nelore de dois, três e quatro anos, em Unaí - MG, submetidos à classificação andrológica e avaliação da libido, com posterior teste de fertilidade a campo (em lotes de 20 e 30 fêmeas/touro) Vale Filho (1994) verificaram que se a pontuação na classificação é pelo menos razoável (acima de 60 pontos) e a libido alta, ou se a libido é razoável e a classificação é alta, a taxa de fecundação é alta, mostrando a importância da avaliação dos touros antes do início da estação de monta.

Costa e Silva (1994) desafiando a fertilidade de touros Nelore, nas condições do pantanal sul matogrossense, selecionados por exame andrológico e teste de libido em acasalamento com fêmeas de diferenças categorias, em estação de monta de 90 dias, nas proporções de 1:40, 1:60 e 1:80, registrou resultados de taxa de gestação de, respectivamente, 93,75\%,76,15\% e 81,25\%. Apesar dos dados ainda não serem conclusivos, a autora relatou que a razão touro:vaca, até onde foi testada, não se constituiu em fator limitante para a fertilidade, mas sim, a condição corporal das fêmeas. Ainda submetendo touros Nelore com alta classificação andrológica e alta libido aos acasalamentos individualizados, na proporção touro:vaca de 1:92, Pineda et al. (1997) obtiveram taxas médias de prenhez de 54,7, 73,8 e 87,0\%, respectivamente, aos 21, 42 e 63 dias de estação de monta. Já Fonseca et al. (1997), utilizando touros Nelore em acasalamentos múltiplos, nas proporções de 1:40 e 1:60, em estação de monta de 120 dias, registraram taxas de gestação aos 30, 60, 90 e 120 dias, respectivamente, de 69,4; 80,5; 88,3 e 89,9\%. Os autores consideram que estes dados desfazem dúvidas de pesquisas anteriores, sobre a influência da hierarquia em acasalamentos múltiplos como fator prejudicial à fertilidade. 
Ressalta-se que, nesta pesquisa foi usada apenas fêmeas solteiras, o que resultou em grande concentração de cios nos primeiros dias da estação de monta, proporcionando grande desafio para os touros. Sugerindo-se que, em lotes com cria ao pé ou mistos, a razão touro:vaca utilizada poderá ser ainda maior, em função da distribuição mais uniforme dos cios ao longo da estação de monta. Ainda segundo Fonseca (2000), reprodutores Nelore bem selecionados por meio de exames andrológicos e comportamentais podem perfeitamente suportar a proporção de 80 vacas por touro, em acasalamentos coletivos em estação de monta de 90 dias, com indiscutíveis vantagens econômicas.

É importante ressaltar que o número de fêmeas servidas por touro numa estação de monta, dependerá, além do seu potencial reprodutivo, de outros fatores tais como: topografia, tamanho das invernadas, condição nutricional dos animais, categoria das fêmeas e práticas de manejo utilizadas na fazenda. Entre as práticas de manejo que poderão ser utilizadas durante a estação de monta, objetivando maior eficiência reprodutiva, a movimentação rotineira do rebanho (rodeio) é fundamental para o estabelecimento de maior razão touro:vaca, principalmente nos manejos extensivos das grandes invernadas. Está prática tem como objetivo uniformizar os touros nos lotes de fêmeas, evitando-se grupos isolados na mesma pastagem (Minosso et al., 2014).

Santos et al. (2003) e Santos et al. (2004) estudando a influência da libido de touros sobre as taxas de gestação de vacas em estação de monta com razão touro:vaca de até 1:75 e 1:100 não se observaram efeito de correlação entre libido, qualidade seminal, circunferência escrotal e concentração sérica de testosterona. Já Lopes et al. (2009) não observaram correlação entre CAP e taxa de gestação, nem entre libido e CAP com taxa geral de gestação após o período de estação de monta de 74,69\%. Esses trabalhos sugerem que os touros Nelore não foram ainda testados em seu limite de capacidade de reprodução na monta a campo, mesmo na razão touro:vaca de 1:100, fator que pode ter contribuído para as baixas correlação entre a CAP, Libido e a taxas de gestação. Franco et al. (2006) corrobora com essa afirmativa ao relatar bons índices de gestação em touros Nelore desafiados a campo na proporção de 1:100, concluindo que reprodutores Nelore selecionados por meio de exames andrológicos e comportamentais (teste da libido) podem suportar até 100 vacas em estação de monta curta, com vantagens econômicas e para a eficiência reprodutiva atual e futura do rebanho, desde que observadas as regras de manejo préestabelecidas.

Em resumo, os vários trabalhos citados com touros das raças taurinas (Neville Júnior et al., 1988; Rupp et al., 1977; Smith et al., 1981) e/ou zebuínos (Costa e Silva, 1994; Crudeli, 1990; Fonseca, 2000; Fonseca et al., 1997; Pineda et al., 2000; Pineda et al., 1997; Santos et al., 2004; Vale Filho, 1994) têm encontrado resultados por vezes contraditórios ou inconclusivos, alguns nem sempre favoráveis aos touros com alta classificação nos exames andrológicos e testes comportamentais, utilizados com diferentes razões touro:vaca, sugerindo que tais relações, bem como a inadequação de alguns dos testes utilizados, juntamente com o efeito da condição corporal das fêmeas, possam estar afetando o percentual de fêmeas gestantes ao final da estação de monta. Já outros autores (Franco et al., 2006; Freneau et al., 2017; Salvador et al., 2003; Salvador et al., 2008a; Salvador et al., 2005; Salvador et al., 2001; Salvador et al., 2008b) lograram associações positivas entre parâmetros andrológicos da CAP e aspectos reprodutivos tais como congelação do sêmen, reação acrossômica induzida e taxa de gestação a campo.

O objetivo deste estudo foi relatar o desempenho reprodutivo de touros da raça Nelore, submetidos à classificação andrológica por pontos (CAP) e ao teste de libido mediante desafio de acasalamento com alto número de fêmeas com estro sincronizado, para que os touros de alta classificação pudessem ser testados em seu limite de potencial de fecundação diária na monta a campo.

\section{Material e métodos}

\section{Descrição do local e pastos do experimento}

$\mathrm{O}$ experimento foi realizado em fazenda de pecuária extensiva do estado do Mato Grosso do Sul (MS), região do pantanal brasileiro, com área total de aproximadamente 5.000 hectares, divididos em pastos com área variável de 25 a 80 hectares, formados na sua maioria por capim Tanzânia, colonião e Braquiária decumbens. O retiro se destina ao manuseio de touros fora do período de estação de monta, recria de tourinhos e manejo de novilhas em inseminação artificial durante o período de estação de monta. 


\section{Testes de fertilidade}

Dentre os 38 touros pré-selecionados, foram escolhidos oito animais de acordo com a classificação andrológica por pontos (CAP) e resultados no teste de libido (Salvador et al., 2008a), os quais foram desafiados quanto a fertilidade, em acasalamentos individualizados, por um período de quatro dias, frente a uma grande proporção de fêmeas em estro sincronizado por protocolo hormonal. Para os testes de fertilidade a campo, foram estabelecidas quatro categorias de touros, com duas repetições cada:

$\mathrm{T} 1=\mathrm{CAP}>75$ pontos e alta libido;

$\mathrm{T} 2=\mathrm{CAP}<75$ pontos e alta libido;

$\mathrm{T} 3=\mathrm{CAP}>75$ pontos e baixa libido;

$\mathrm{T} 4=\mathrm{CAP}<75$ pontos e baixa libido

Os touros passaram por período de adaptação ao buçal marcador, com duração de 10 dias anterior ao período de acasalamento e foram introduzidos nos lotes no dia da retirada dos implantes das fêmeas, permanecendo junto às mesmas por período de quatro dias. Os pastos dos oito lotes do teste de fertilidade variavam entre 25 a 60 hectares e os animais foram submetidos ao desafio em dois períodos subsequentes intervalados de dois dias. Foram tomados os cuidados de testar um touro de cada tratamento na primeira e na segunda bateria de testes, bem como de não se usar o mesmo tipo de touro em um mesmo pasto, para que não houvesse a possibilidade de interferência dos efeitos de baterias de touros e dos pastos utilizados nos índices de fertilidade.

\section{Lotes de Fêmeas}

Utilizaram-se de 43 a 45 fêmeas Nelore por lote (34 com implante e 10 sem implante), totalizando 352 fêmeas (173 novilhas e 179 vacas solteiras) com idades variando de três a oito anos, avaliadas ginecologicamente e distribuídas uniformemente pelos tratamentos, de acordo com a condição ovariana, escore corporal, idade e dia aplicação do implante. Diante da necessidade de se realizar duas baterias de teste com os touros, metade do total de fêmeas foi sincronizada seis dias após a primeira sincronização, para que os touros da bateria subsequente tivessem as mesmas condições de fêmeas sincronizadas, que os touros da primeira bateira. Os cios distribuíram-se entre 18 e 78 horas, com picos entre 42 e 54 horas, após a retirada dos implantes, resultando, em média, 24,6 $\pm 2,11$ fêmeas em cio por touro, em acasalamento individual.

\section{Período de observação}

A real proporção de fêmeas em cio por touro em cada lote foi verificada em dois rodeios de 40 minutos, feitos pela manhã e tarde, de 12 até 96 horas após a retirada dos implantes, para a verificação das fêmeas em cio ou envolvidas no grupo com atividade sexual. Durante os períodos de rodeio, também foram feitas anotações dos comportamentos avaliados no teste de libido, realizados pelo touro, o que foi considerado como comportamento a campo destes animais. Estes dados foram correlacionados com a classificação quando do teste de libido realizado em curral.

\section{Coletas dos resultados e análises estatísticas}

O diagnóstico de gestação foi realizado por palpação retal, 60 dias após o encerramento do período do estudo. Após os quatro dias de desafio dos touros, as fêmeas usadas ficaram separadas dos touros por período de 30 dias, para então serem novamente entouradas no repasse. As análises das taxas de prenhez, por categoria de touro, foram realizadas pelo método de Qui-quadrado $(\mathrm{P}<0,05)$ de acordo com Sampaio (1998).

\section{Resultados e discussão}

\section{Sincronização dos cios e mensuração do desafio de fertilidade}

As manifestações de cios foram observadas no período de 18 a 78 horas após a retirada dos implantes. Os maiores percentuais de manifestação de cios foram registrados às $42(40,1 \%)$ e às 54 $(30,4 \%)$ horas após a remoção dos implantes. Observou-se uma distribuição uniforme dos cios por todos 
os lotes de fêmeas, condição essencial para realizar as comparações dos desempenhos reprodutivos dos touros testados. O número de fêmeas em cio durante os quatro dias de desafio, variou de 22 a 29 , com média de 24,6 $\pm 2,11$ em cio por lote de touro testado.

É importante salientar que, apesar das medidas de observação dos cios (rodeios e buçal marcador), a identificação de animais em cio não foi totalmente adequada, podendo ter ocorrido falhas na detecção do estro de algumas fêmeas, provavelmente devido a cios atípicos (de curta duração e/ou silencioso), comumente observados em fêmeas sincronizadas por protocolos de indução hormonal. Este fato é confirmado pela presença de nove animais do total de fêmeas em monta, que apesar de não terem sido confirmadas em cio durante os rodeios, se apresentaram gestantes ao diagnóstico de gestação, com o número de dias de gestação coincidente com a época de cobertura dos touros.

Na tabela 1 são apresentadas as taxas de detecção de estros dos oito lotes de fêmeas sincronizadas, onde não foram constatadas diferenças $(\mathrm{P}>0,05)$ entre os mesmos, mostrando adequada condição de igualdade para touros nos testes de desafio a campo.

Tabela 1. Número e taxas de detecção de estro dos oito lotes de fêmeas, usadas durante os testes de fertilidade a campo dos touros Nelore, sincronizadas previamente.

\begin{tabular}{|c|c|c|c|}
\hline Lote & N. ${ }^{o}$ de fêmeas em monta & $\begin{array}{l}\mathrm{N}^{\circ} \text { de fêmeas observadas em } \\
\text { estro nos quatro dias de desafio }\end{array}$ & $\begin{array}{l}\text { Taxas de detecção } \\
\text { de estro, } \%\end{array}$ \\
\hline 1 (alta CAP, alto libido) & 43 & 22 & 51,16 \\
\hline 2 (baixa CAP, baixo libido) & 44 & 24 & 54,54 \\
\hline 3 (alta CAP, baixo libido) & 44 & 25 & 56,81 \\
\hline 4 (baixa CAP, alto libido) & 44 & 29 & 65,90 \\
\hline 5 (alta CAP, baixo libido) & 43 & 26 & 60,46 \\
\hline 6 (baixa CAP, alto libido) & 44 & 22 & 50,00 \\
\hline 7 (alta CAP, alto libido) & 44 & 24 & 54,54 \\
\hline 8 (baixa CAP, baixo libido) & 45 & 25 & 55,55 \\
\hline
\end{tabular}

$\mathrm{P}>0,05$, Teste $\chi^{2}$.

O número médio de fêmeas em cio por touro, durante os quatro dias de desafio a campo, foi aparentemente superior ao potencial de fecundação diária, mesmo considerando touros de alta libido e CAP, desafiados por curto período de tempo. A partir dos dados da tabela 1, pode-se estimar uma média de seis fêmeas em cio por touro em cada lote do teste de desafio a campo. No entanto, durante o pico de manifestação de cios, foi registrado um desafio de monta de 9,87 $\pm 1,55$ fêmeas em cio por touro. Segundo Galloway (1989), este número excede em muito o potencial de produção espermática e fecundação mesmo para touros com CE acima de $35 \mathrm{~cm}$.

Em uma estação de monta de noventa dias, touros que estivessem servindo em uma razão touro:vaca de 1:75 teriam um desafio diário variando de 2,5 a 0,42 fêmeas em cio, do início para o final da estação. Estas estimativas levam também à ideia de que o desafio diário, em que estes animais foram submetidos foi muito superior ao de outros trabalhos descritos na literatura usando-se razões touro:vaca variáveis de 1:40 a 1:80 (Costa e Silva, 1994; Fonseca, 2000; Fonseca et al., 1997; Pineda et al., 2000).

\section{Características dos touros selecionados}

Dos 38 touros reavaliados andrologicamente e submetidos ao teste de libido, foram selecionados quatro animais com CAP acima de 75 pontos e quatro com CAP abaixo de 75 pontos para o desafio a campo, com distribuição uniforme quanto à classificação no teste de libido, cujas médias são comparadas na tabela 2. Observa-se que a diferenciação dos touros de alta e baixa CAP deveram-se principalmente, aqueles relacionados aos aspectos físicos do sêmen $(\mathrm{P}<0,05)$ e a $\mathrm{CE}$ que, apesar de não apresentar diferença $(\mathrm{P}>0,05)$, em função do reduzido número de animais, apresentou tendência favorável aos touros de mais alta CAP. Tais diferenças na CE e nos aspectos físicos do sêmen poderiam comprometer a taxa de fecundação, somente diante de alto desafio de fêmeas em cio, onde possivelmente a capacidade de produção espermática diária seria insuficiente para fecundar um grande número diário de fêmeas, conforme observações de Galloway (1989). 
Tabela 2. Médias de pesos, circunferências escrotais (CE) e características seminais de oito touros adultos da raça Nelore, selecionados para o desafio de fertilidade a campo.

\begin{tabular}{lccccccc}
\hline $\begin{array}{l}\text { Categoria de } \\
\text { CAP }\end{array}$ & CE $(\mathrm{Cm})$ & Motilidade (\%) & $\begin{array}{c}\text { Vigor } \\
(1-5)\end{array}$ & $\begin{array}{c}\text { Concentração } \\
\text { espermática } \\
(\text { Sptz x 10 })\end{array}$ & $\begin{array}{c}\text { Defeitos } \\
\text { maiores }(\%)\end{array}$ & $\begin{array}{c}\text { Defeitos totais } \\
(\%)\end{array}$ & $\begin{array}{c}\text { CAP } \\
(0-100 \text { pontos })\end{array}$ \\
\hline$>75$ & $37,6 \pm 0,47$ & $62,5 \pm 2,88 \mathrm{a}$ & $3,7 \pm 0,5 \mathrm{a}$ & $672,5 \pm 432,4$ & $6,5 \pm 1,73$ & $12,5 \pm 1,73$ & $76,0 \pm 0,00 \mathrm{a}$ \\
$<75$ & $34,1 \pm 1,10$ & $51,2 \pm 7,5 \mathrm{~b}$ & $2,2 \pm 0,95 \mathrm{~b}$ & $329,7 \pm 45,50$ & $4,7 \pm 2,36$ & $15,0 \pm 4,08$ & $63,5 \pm 9.03 \mathrm{~b}$ \\
\hline
\end{tabular}

$\mathrm{P}<0,05$, Teste SNK.

\section{Desafio de fertilidade}

Na tabela 3 são mostradas as taxas de gestação em relação ao total de fêmeas acasaladas com os touros durante os quatro dias de desafio de fertilidade. Os dados foram processados de forma que a fertilidade foi obtida pelo somatório dos dois touros de cada um dos quatro tratamentos.

Tabela 3. Número e taxas de gestação de todas as fêmeas em monta, obtidas em quatro dias, por touros da raça Nelore, com diferente libido e classificação andrológica por pontos (CAP), quando desafiados com alta pressão de fêmeas previamente sincronizadas.

\begin{tabular}{|c|c|c|c|}
\hline Tratamentos & N. ${ }^{o}$ de fêmeas em monta & N. ${ }^{\circ}$ de fêmeas gestantes & Taxas de gestação, $\%$ \\
\hline T1: CAP > 75 pontos e alta libido & 87 & 26 & $29,88 \mathrm{a}$ \\
\hline T2: CAP $<75$ pontos e alta libido & 86 & 18 & $20,93 \mathrm{ab}$ \\
\hline T3: CAP > 75 pontos e baixa libido & 87 & 16 & $18,39 \mathrm{ab}$ \\
\hline T4: CAP $<75$ pontos e baixa libido & 89 & 14 & $15,73 \mathrm{~b}$ \\
\hline Geral & 349 & 74 & 21,20 \\
\hline
\end{tabular}

$\mathrm{P}<0,05$, Teste $\chi^{2}$.

Observa-se que os dois touros do T1 fecundaram um total de 26 fêmeas, num período de quatro dias, o que corresponde, em média, aproximadamente a seis fêmeas servidas e fecundadas por dia, e sugere a possibilidade de utilização de uma maior proporção touro:vaca, mesmo em estações de monta fixas e limitadas. Ressalta-se que os resultados de taxas de gestações aqui observadas não podem ser extrapolados integralmente para desafios de monta a campo, uma vez que o desgaste do touro seria muito grande. Além disto, o número de fêmeas em cio obtido pela sincronização foi aparentemente excessivo para o potencial de fecundação diária dos touros, mesmo considerando o curto período de desafio. O número médio geral de fêmeas gestante por touro foi de $9,25 \pm 3,2$, durante os quatro dias de acasalamento, sendo este resultado bastante superior aos observados por Smith et al. (1981) para touros jovens Santa Gertrudis, que registraram 4,7 \pm 3,6 fêmeas gestantes por touro, trabalhando também com desafio de acasalamento de quatro dias; porém, com média inferior de fêmeas em estro sincronizado por touro $(17,6 \pm 3,5)$, variando de 12 a 27.

Na tabela 4 são apresentadas as taxas de gestação ao final dos quatro dias dos testes de fertilidade, em função da quantidade de fêmeas observadas em cio, que variaram de 22,44 a 47,82\%, para T4 e T1 $(\mathrm{P}<0,05)$, respectivamente. Foram observados valores intermediários para o T2 e T3. Não se registraram, porém, diferenças $(\mathrm{P}>0,05)$ entre os percentuais de vacas gestantes para os grupos $\mathrm{T} 2, \mathrm{~T} 3$ e T4, bem como para T1, T2 e T3, embora o T1 apresentasse, aproximadamente, 20\% a mais de fêmeas gestantes em relação a T3. Observou-se que a taxa de fêmeas gestantes dos touros do T1 (Alto CAP e alto libido) foi $47,82 \%$, semelhante a alguns trabalhos descritos na literatura para taxa de prenhez à primeira cobrição (Fonseca et al., 1997) e inferior aos resultados relatados por vários outros autores (Costa e Silva, 1994; Fonseca, 2000; Freneau et al., 2017; Pineda et al., 1997; Santos et al., 2004) trabalhando com razões touro:vaca variáveis de 1:40 a 1:80.

Já a taxa geral de fêmeas gestantes, registradas para o grupo de fêmeas observadas em estro, neste estudo, foi de 33,3\%, considerada satisfatória, quando comparada com os resultados de Smith et al. (1981) trabalhando em condições já descritas anteriormente. Os referidos autores observaram taxa geral de prenhez das fêmeas em estro de $26,8 \%$, concluindo que estes resultados foram possivelmente devido à combinação do curto período de cobrição e a utilização de grande número de touros com qualidade espermática fraca e falta de experiência sexual prévia. 
Tabela 4. Número e taxas de gestação das fêmeas observadas em estro, obtidas em quatro dias, por touros da raça Nelore, com diferentes libidos e classificação andrológica por pontos (CAP), quando desafiados com alta pressão de fêmeas previamente sincronizadas.

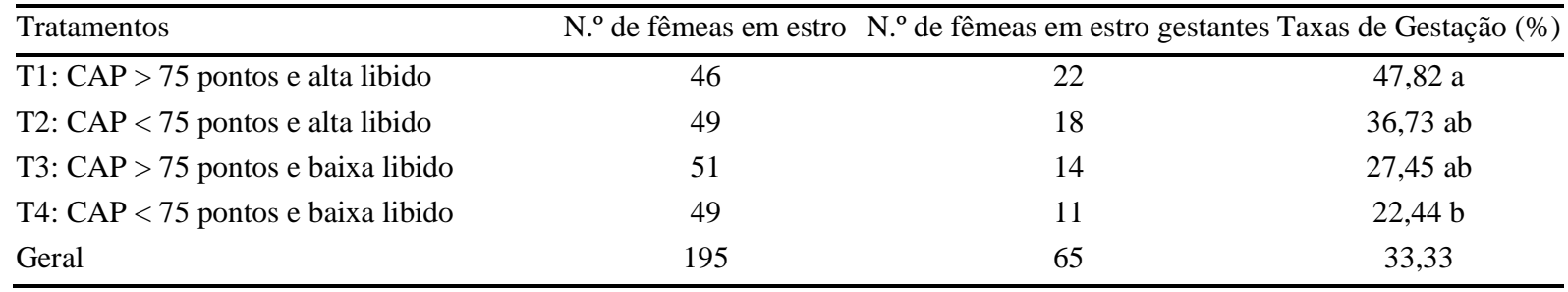

$\mathrm{p}<0,05$, Teste $\chi^{2}$.

Analisando-se os resultados de taxa de fertilidade, com os touros agrupados em baixa (CAP $<75$ pontos) e alta (CAP > 75 pontos) classificação andrológica por pontos (CAP) e considerando-se uma distribuição uniforme na classificação da libido, observa-se que não houve diferença $(\mathrm{P}>0,05)$ entre os touros, apesar de se observar maior taxa de fertilidade nos touros de alta CAP (Tabela 5).

Tabela 5. Número e taxas de gestação de todas as fêmeas em monta, obtidas em quatro dias, por touros da raça Nelore, com classificação andrológica por pontos acima e abaixo de 75 pontos, quando desafiados com alta pressão de fêmeas previamente sincronizadas.

\begin{tabular}{lccc}
\hline Classificação andrológica por pontos & N. $^{\text {o de fêmeas em estro }}$ & N. $^{\text {o de fêmeas gestantes }}$ & Taxas de gestação (\%) \\
\hline$>75$ & 97 & 36 & 37,11 \\
$<75$ & 98 & 29 & 29,59 \\
\hline
\end{tabular}

$\mathrm{P}>0,05$, Teste $\chi^{2}$.

Quando foram analisados os resultados da taxa de fertilidade, com os touros agrupados de acordo com a libido, considerando-se uniforme a classificação pela CAP, observou-se que houve diferença (P $<0,05)$ entre os touros de alta e baixo libido.

Estas análises são indicativas de que, nas condições deste experimento, com alto desafio de fêmeas em cio por touro em acasalamento individualizado, a libido exerceu maior influência nas taxas de fertilidade do que as diferenças alcançadas na pontuação dos touros com CAP acima de 60 pontos. Estes resultados concordam com aqueles relatados por vários autores (Blockey, 1978; Pineda et al., 2000; Smith et al., 1981); porém, discordam dos resultados de Farin et al. (1989) que observaram que touros de maior libido, serviam maior número de fêmeas, sem, contudo encontrar correlação com à taxa de prenhez.

Na tabela 6 são apresentadas as taxas de gestação em função do horário de manifestação dos cios. Observou-se que as taxas mais altas de gestação foram registradas nos períodos de até 30 horas $(34,6 \%)$ e com 42 horas $(42,7 \%)$ após a retirada os implantes. Observou-se ainda decréscimo nas taxas de gestação das fêmeas que manifestaram cio nos períodos de 54 e após as 66 horas da remoção do implante. Este fato pode ser explicado em função da menor fertilidade das fêmeas que manifestaram cio 48 horas após a remoção dos implantes, conforme descrito por Quirino et al. (2001), possivelmente em função da queda de fertilidade observada nos protocolos de sincronização após este período, aliada à exaustão dos touros, que foram excessivamente requeridos no momento do pico de manifestações dos cios.

Observou-se que, embora fosse constatada diminuição geral da fertilidade às 54 horas após a retirada dos implantes, os touros do T1 (alta CAP e alto libido) mantiveram maior taxa de prenhez em relação aos demais tratamentos. Observou-se ainda tendência de aumento da taxa de prenhez no T2 (CAP < 75 e alta libido) no período do pico principal de manifestação dos cios, onde o desafio de cobrição foi de $9,87 \pm 1,55$ fêmeas em cio por touro. Este resultado pode ser afetado pelo fator limitante para a fecundação neste momento ter sido, provavelmente, à capacidade de monta dos touros, onde a libido exerceu maior importância que as diferenças relacionadas aos aspectos qualidade e produção espermática diária dos touros selecionados pela CAP. Posteriormente, ao período de pico dos estros, os touros do T2 apresentaram as menores taxas de gestações entre os tratamentos, o que pode ser justificado pelo desgaste físico durante o pico de cios, sugerindo diminuição do número de montas completas. Outra hipótese seria o esgotamento destes touros em termos de capacidade de produção espermática diária no 
pico de cios, em virtude de serem touros de baixa CAP, não conseguindo se manter com alto potencial de fecundação durante um período mais prolongado, conforme descrito anteriormente.

Ressalta-se, no entanto, que não foram registradas diferenças $(\mathrm{P}>0,05)$ na comparação entre os tratamentos, possivelmente pelo número reduzido de fêmeas em cada categoria de horários de manifestação de cios.

Tabela 6. Taxas de gestação das fêmeas em função do horário de manifestação dos cios, obtidas por touros Nelore, com diferentes libidos e classificação andrológica por pontos (CAP), desafiados com alta pressão de fêmeas previamente sincronizadas

\begin{tabular}{lcccc}
\hline \multirow{2}{*}{ Tratamentos } & \multicolumn{3}{c}{ Taxas de gestação (\%) das fêmeas que manifestaram cio em: } \\
\cline { 2 - 5 } & Até 30h & $42 \mathrm{~h}$ & $54 \mathrm{~h}$ & Acima 66 h \\
\hline T1: CAP > 75 pontos e alta libido & 50,00 & 50,00 & 38,46 & 66,67 \\
T2: CAP < 75 pontos e alta libido & 40,00 & 52,38 & 14,29 & 30,00 \\
T3: CAP > 75 pontos e baixa libido & 33,33 & 33,33 & 26,67 & 22,22 \\
T4: CAP < 75 pontos e baixa libido & 0,00 & 29,41 & 26,67 & 22,22 \\
Geral & 34,62 & 42,67 & 26,32 & 29,03 \\
\hline
\end{tabular}

$\mathrm{p}>0,05$, Teste $\chi^{2}$ fisher.

\section{Conclusões}

Em animais previamente selecionados, o teste de libido exerceu maior influência nas taxas de gestações finais que a pontuação atingida na CAP, quando os touros foram desafiados com elevado número de fêmeas em cio, sugerindo que a capacidade de monta diária seja o principal fator limitante na obtenção de altas taxas reprodutivas. A seleção de touros com elevadas pontuações na CAP, e consequentemente maior quantidade e qualidade espermática, mostrou-se eficiente na manutenção das taxas de gestação durante o período de alto desafio de monta.

$\mathrm{O}$ alto percentual de fêmeas em cio previamente sincronizadas, permitiu identificar o real potencial reprodutivo e de fecundação de touros Nelore, previamente avaliados quanto aos aspectos andrológicos e de comportamento sexual, sugerindo que touros adultos com alta CAP e alto libido poderão ser utilizados com uma razão touro:vaca superior a comumente utilizada na maioria dos rebanhos (1:40), sem comprometimento da taxa de gestação ao final da estação de monta. O limite superior para touros de alto desempenho reprodutivo para CAP e libido parece ser o de 8 a 10 fêmeas gestantes em períodos de acasalamento de quatro dias. Em condições extensivas de monta, isso seria equivalente a uma razão touro:vaca de mais de 1:200, proporção não relatada na literatura para qualquer raça bovina. Ressaltamos que as condições nutricionais (score corporal) teriam que ser mantidas sobre constante controle para que a capacidade de cobertura e fecundação desses touros de alto desempenho quanto a CAP e libido fossem mantidas durante todo o período da estação de monta.

\section{Referências bibliográficas}

Blockey, M. A. B. (1978). The influence of serving capacity of bulls on herd fertility. Journal of Animal Science, 46(3):589-595.

Chenoweth, P. J. (1983). Sexual Behavior of the Bull: A Review. Journal of Dairy Science, 66(1):173179.

Chenoweth, P. J., Farin, P. W., Mateos, E. R., Rupp, G. P. \& Pexton, J. E. (1984). Breeding soundness and sex drive by breed and age in beef bulls used for natural mating. Theriogenology, 22(4):341-349.

Costa e Silva, V. E. (1994). Avaliação da capacidade reprodutiva de touros Nelore. Exame andrológico, teste de comportamento sexual e desafio da fertilidade. Master of Science, Universidade Federal de Minas Gerais, Belo Horizonte, Minas Gerais, Brasil.

Crudeli, G. A. (1990). Avaliação da aptidão reprodutiva de touros de raça Nelore e seu efeito sobre a taxa de gestação do rebanho. Master of Science, Universidade Federal de Minas Gerais, Belo Horizonte, Minas Gerais, Brasil.

Farin, P. W., Chenoweth, P. J., Tomky, D. F., Ball, L. \& Pexton, J. E. (1989). Breeding soundness, libido and performance of beef bulls mated to estrus synchronized females. Theriogenology, 32(5):717-725. 
Fonseca, V. O. (2000). O touro no contexto da eficiência reprodutiva do rebanho. Informe Agropecuário, 21(205):48-63.

Fonseca, V. O., Franco, C. S., Bergmann, J. A. G., Chow, L. A. \& Assumpção, T. I. (1997). Potencial reprodutivo de touros da raça Nelore (Bos taurus indicus) acasalados com elevado número de vacas. Arquivo Brasileiro de Medecina Veterinária e Zootecnia, 49(1):53-62.

Franco, C. S., Fonseca, V. O. \& Gaste, L. (2006). Potencial reprodutivo de touros Nelore acasalados coletivamente na proporção de um touro para 100 vacas. Arquivo Brasileiro de Medecina Veterinária e Zootecnia, 58(6):1156-1161.

Freneau, G. E., Puoli, J. R. \& Arnhold, E. (2017). Evolução após quatro anos de aplicação da capacidade andrológica por pontos (CAP) em touros nelore e estudo da relação com a prenhez em estação de acasalamento. Ciência Animal Brasileira, 18(e-31274, 2017):1-12.

Galloway, D. B. (1989). Factors affecting fertility in bulls. Revista brasileira de Reprodução Animal, $127-46$.

Lopes, F. G., Guimarães, J. D., Costa, E. P., Carvalho, G. R. \& Miranda Neto, T. (2009). Avaliação andrológica por pontos e comportamento sexual em touros da raça Nelore. Revista Brasileira de Zootecnia, 38(6):1018-1025.

Minosso, L. A., Santos, M. D., Toma, H. S., Toma, C. D. M., Costa, D. S., Junior, D. A. F. \& Lopes, F. G. (2014). Manejo de rodeio diário como ferramenta para incrementar a taxa de prenhes de vacas Nelore. Revista Brasileira de Higiene e Sanidade Animal: RBHSA, 8(2):39-51.

Neville Júnior, W. E., Williams III, D. J., Richardson, K. L. \& Utley, P. R. (1988). Relationship of breeding soundness evaluation score and its components with reproductive performance of beef bulls. Theriogenology, 30(3):429-436.

Pineda, N. R., Fonseca, V. O. \& Albuquerque, L. G. (2000). Estudo preliminar da influência do perímetro escrotal sobre a libido em touros jovens da raça Nelore. Arquivo Brasileiro de Medicina Veterinaria e Zootecnia, 52(1):69-75.

Pineda, N. R., Lemos, P. F. \& Fonseca, V. O. (1997). Comparação entre dois testes de avaliação do comportamento sexual (libido) de touros Nelore (Bos taurus indicus). Revista Brasileira de Reprodução Animal, 21(4):29-34.

Quirino, C. R., Salvador, D. F., Andrade, V. J., Vale Filho, V. R., Ribeiro Filho, A. L., Nogueira, L. A. G., . . Gattass, C. (2001). Eficiencia reprodutiva de femeas Nelore sincronizadas com o protocolo Crestar, acasaladas com touros previamente selecionados pela classificacao andrológica por pontos (CAP) e libido. Revista Brasileir de Reproduçõ Animal, 25(3):304-307.

Rupp, G. P., Ball, L., Shoop, M. C. \& Chenoweth, P. J. (1977). Reproductive efficiency of bulls in natural service: effects of male to female ratio and single-vs multiple-sire breeding groups. Journal of the American Veterinary Medical Association, 171(7):639-642.

Salvador, D., Andrade, V., Vale Filho, V., Silva, A. \& Costa e Silva, E. (2003). Avaliação da libido de touros Nelore adultos em curral e sua associação com características andrológicas e desempenho reprodutivo a campo. Arquivo Brasileiro de Medicina Veterinaria e Zootecnia, 55(5):588-593.

Salvador, D. F., Andrade, V. J., Nogueira, L. A. G. \& Vale Filho, V. R. (2008a). Avaliação dos eventos sexuais de touros Nelore adultos em teste de libido realizado em curral. Revista Brasileira de Ciência Veterinária, 15(1):15-20.

Salvador, D. F., Andrade, V. J., Nogueira, L. A. G., Vale Filho, V. R., Santoro, M. M., Folhadela, I. \& Salvador, R. R. S. (2005). Association among andrologic selection, semen freezing and induction of acrossome reactions by heparin in two-year Nelore bulls. Reproduction in Domestic Animals, 4363.

Salvador, D. F., Andrade, V. J. \& Vale Filho, V. R. (2001). Desempenho reprodutivo de touros da raça Nelore, submetidos à classificação andrológica por pontos (CAP), à libido e desafiados com alto número de fêmeas com estro sincronizado. Revista Brasileira de Reprodução Animal, 25(2):185187.

Salvador, D. F., Andrade, V. J., Vale Filho, V. R., Dias, J. C. \& Nogueira, L. A. G. (2008b). Associação entre o perfil andrológico e a congelação de sêmen de touros da raça Nelore aos dois anos de idade, 
pré-selecionados pela classificação andrológica por pontos (CAP). Arquivo Brasileiro de Medicina Veterinária e Zootecnia, 60(3):587-593.

Sampaio, I. B. M. (1998). Estatística aplicada à experimentação animal (Vol. 1). Belo Horizonte, Minas Gerais, Brasil: FEP-MVZ.

Santos, M. D., Torres, C. A. A., Guimarães, J. D., Ruas, J. R. M. \& Carvalho, G. R. (2003). Libido de touros Nelore: efeito da proporção touro: vaca sobre a taxa de gestação. Arquivo Brasileiro de Medicina Veterinária e Zootecnia, 55(3):293-300.

Santos, M. D., Torres, C. A. A., Ruas, J. R. M., Silva Filho, J. M., Costa, E. P. \& Pereira, J. C. (2004). Teste da libido e atividade de monta em touros da raça Nelore. Arquivo Brasileiro de Medicina Veterinária e Zootecnia, 56(4):504-510.

Smith, M. F., Morris, D. L., Amoss, M. S., Parish, N. R., Williams, J. D. \& Wiltbank, J. N. (1981). Relationships among fertility, scrotal circumference, seminal quality, and libido in Santa Gertrudis bulls. Theriogenology, 16(4):379-397.

Vale Filho, V. R. (1988). Desenvolvimento testicular em touros: aspectos clínicos. Paper presented at the Congresso Brasileiro de Reprodução, Belo Horizonte, Minas Gerais, Brasil.

Vale Filho, V. R. (1994). Seleção de touros e matrizes, em estação de monta, para alta eficiência reprodutiva. Paper presented at the ABCZ, Uberaba, Minas Gerais, Brasil.

Vale Filho, V. R., Quirino, C. R., Andrade, V. J., Bergmann, J. A. G., Reis, S. R., Mendonça, R. M. A. \& Fonseca, C. G. (1999). Parâmetros genéticos da classificação andrológica por pontos (CAP), em touros da raça Nelore. Revista Brasileira de Reprodução Animal, 23253-255.

Recebido: 16 de setembro, 2019.

Aprovado: 14 de outubro, 2019.

Publicado: 25 de outubro, 2019.

Licenciamento: Este artigo é publicado na modalidade Acesso Aberto sob a licença Creative Commons Atribuição 4.0 (CC-BY 4.0), a qual permite uso irrestrito, distribuição, reprodução em qualquer meio, desde que o autor e a fonte sejam devidamente creditados. 